Chirurg 2012 $\cdot 83: 917$

DOI 10.1007/s00104-012-2366-y

Online publiziert: 26 . September 2012

(c) Springer-Verlag Berlin Heidelberg 2012

J. Reibetanz • C.-T. Germer

Klinik für Allgemein-, Viszeral-, Gefäß- und Kinderchirurgie, Universitätsklinikum Würzburg

\title{
Technische Machbarkeit und Sicherheit der Single-Port-Kolonchirurgie
}

\section{Ergebnisse}

Dreiundzwanzig Studien mit insgesamt 378 Patienten wurden in die Metaanalyse inkludiert, wobei bei der überwiegenden Mehrzahl der Fälle eine Hemikolektomie rechts erfolgte $(n=279)$. Alle außer 3 Studien schlossen Patienten mit Kolonkarzinomen mit ein, in allen bis auf 2 Studien wurden kommerziell erhältliche Single-Port-Trokarsysteme verwendet. Bei Operationszeiten zwischen 83 und 225 min zeigte sich eine vergleichsweise niedrige Konversionsrate von insgesamt $6,9 \%$ (1,6\% offen, $1,6 \%$ handassistiert, $4 \%$ Multi-Port-Laparoskopie). Bei weiteren $4,9 \%$ der Patienten musste ein zusätzlicher Trokar platziert werden. In Studien, die die SILC-Resektion von Kolonkarzinomen einschlossen, wurden zwischen 13 und 27 Lymphknoten entfernt, histologisch tumorfreie Resektatränder wurden in allen Fällen bestätigt. Die Gesamtletälität bzw. -morbidität betrug 0,5\% (2/378) bzw. 12,9\% (45/349), wobei letztere einer großen Heterogenität zwischen den Studien unterworfen war (0-40\%). Die postoperative stationäre Verweildauer betrug zwischen 1,9 und 9,8 Tagen.

\section{Diskussion und Fazit}

des Reviewers

Anhand des vorliegenden systematischen Reviews werden, bei insgesamt noch geringer Patientenzahl, die generelle technische Machbarkeit und die Sicherheit der SILC-Methode in der Kolonchirurgie bestätigt. Die Kurzzeitergebnisse (Operationszeiten, Morbiditätsra- ten, stationäre Verweildauer) sind vergleichbar mit den bereits publizierten Resultaten der Multi-Port-Laparoskopie. Bei adäquater Lymphknotenzahl und tumorfreien Präparaterändern scheint die Einhaltung onkologischer Standards mit SILC prinzipiell möglich. Allerdings werden im vorliegenden Review, wie auch in den meisten Einzelpublikationen zu SILC, das hochselektionierte Patientengut und die besondere laparoskopische Expertise des Operateurs betont. Dies spiegelt sich nicht zuletzt in der extrem niedrigen Konversionsrate zur offenen Operation von lediglich $1,6 \%$ wider - einem Bruchteil der Konversionsrate verglichen mit der MIC-Kolonchirurgie (!). Zusammenfassend bleibt auch nach diesem Review offen, ob die SILC gegenüber der MIC-Kolonchirurgie einen messbaren Benefit besitzt; dies können nur prospektive Arbeiten zeigen. Hinsichtlich der erhofften kosmetischen Vorteile muss jedoch einschränkend eingeräumt werden, dass die Inzisionlänge bei diesen Eingriffen in der Regel durch die Präparatebergung und weniger durch den verwendeten Port determiniert wird.

\section{Korrespondenzadresse}

Prof. Dr. C.-T. Germer

Klinik für Allgemein-, Viszeral-, Gefäß- und Kinderchirurgie, Universitätsklinikum Würzburg, Oberdürrbacher Str. 6, 97080 Würzburg Germer_c@chirurgie.uni-wuerzburg.de

Interessenkonflikt. Der korrespondierende Autor gibt für sich und seinen Koautor an, dass kein Interessenkonflikt besteht. 